\title{
Bistability in organic magnetic materials. A comparative study of the key differences between hysteretic and non-hysteretic spin transitions in dithiazolyl radicals
}

\author{
Sergi Vela ${ }^{[a, c]}$, Michael B. Reardon ${ }^{[b]}$, Charles E. Jakobsche ${ }^{[b]}$, Mark M. \\ Turnbull $^{[b]}$, Jordi Ribas-Arino*[a] and Juan J. Novoa* ${ }^{*[a]}$
}

${ }^{\left[{ }^{a]}\right.}$ Departament de Ciència de Materials i Química Física, Universitat de Barcelona and IQTCUB, Av. Diagonal 645, 08028 Barcelona (Spain)

${ }^{[b]}$ Carlson School of Chemistry \& Biochemistry, Clark University, 950 Main Street, Worcester, MA 01610, USA

[c] Laboratoire de Chimie Quantique, Institut de Chimie, UMR 7177, CNRSUniversité de Strasbourg, 1 Rue Blaise Pascal, 67000 Strasbourg, France

*j.ribas@ub.edu, juan.novoa@ub.edu 


\section{Abstract}

Dithiazolyl (DTA)-based radicals have furnished many examples of organic spintransition materials, some of them occurring with hysteresis and some others without. Here, we present a combined computational and experimental study aimed at deciphering the factors controlling the existence or absence of hysteresis by comparing the phase transitions of 4-cyanobenzo-1,3,2-dithiazolyl and 1,3,5-trithia-2,4,6triazapentalenyl radicals, which are prototypical examples of non-bistable and bistable spin transitions, respectively. Both materials present low-temperature diamagnetic and high-temperature paramagnetic structures, characterized by dimerized $(\cdots A-A \cdots A-$ $A \cdots)_{n}$ and regular $(\cdots A \cdots A \cdots A \cdot \cdots)_{n}$ m-stacks of radicals respectively. We show that the regular $\pi$-stacks are not potential energy minima but average structures arising from a dynamic interconversion between two degenerate dimerized configurations: $(\cdots A-A \cdots A-A \cdots)_{n} \leftrightarrow(-A \cdots A-A \cdots A-)_{n}$. The emergence of this intrastack dynamics upon heating gives rise to a second-order phase transition that is responsible for the change in the dominant magnetic interactions of the system. This suggests that the promotion of a $(\cdots A-A \cdots A-A \cdots)_{n} \leftrightarrow(-A \cdots A-A \cdots A-)_{n}$ dynamics is a general mechanism for triggering spin transitions in DTA-based materials. Yet, this intrastack dynamics does not suffice to generate bistability, which also requires a rearrangement of the intermolecular bonds between the m-stacks via a first-order phase transition. 


\section{Introduction}

Research in bistable molecule-based magnets (that is, crystals of radicals presenting two polymorphic forms that feature different magnetic properties within a range of temperatures, called the bistability range) has generated a great deal of attention in recent years in view of their potential as nano-devices that could be used as sensors and memory storage units in molecular electronics and spintronics. ${ }^{1,2}$ The achievement of a deep understanding of the features governing the existence of bistability is a necessary step towards the rational design of new bistable materials. Let us mention that bistability is merely a shorter form for naming hysteretic spin-switch systems. In non-hysteretic spin-switch systems, crystals can be obtained in their low-temperature (LT) polymorph or high-temperature (HT) polymorph depending on the temperature, $T$ : (a) above the spin-transition temperature $\left(T_{c}\right)$ (i.e, at $T>T_{c}$ ) all crystals transform into the HT polymorph, and (b) below the spin-transition temperature (i.e, at $T<T_{\mathrm{c}}$ ) all crystals transform into the LT polymorph. However, in hysteretic spin-switch systems, the $\mathrm{LT} \rightarrow \mathrm{HT}$ transition takes place at a different temperature than that of the HT $\rightarrow \mathrm{LT}$ transition. If we denote these two different transition temperatures by $T_{\mathrm{c} \uparrow}$ and $T_{\mathrm{c} \downarrow}$, respectively, there is a range of temperatures comprised between $T_{\mathrm{c} \uparrow}$ and $T_{\mathrm{c} \downarrow}$ (known as the bistability region) in which both the HT and LT phases can exist depending on the history of the system. At $T<T_{\mathrm{c} \downarrow}$, only the LT polymorph exists, while at $T>T_{\mathrm{c} \uparrow}$ only the HT polymorph exists. Bistable materials based on transition-metal complexes have been associated with spin transitions for many years ${ }^{3,4,5}$, but recent work has demonstrated that purely-organic radicals can also be versatile building blocks to achieve bistability $6,7,8,9,10,11,12,13,14,15,16,17$.

Among the purely organic radicals exhibiting spin-switch behavior, the family of dithiazolyl (DTA) neutral radicals ${ }^{18,19}$ is particularly interesting, as it has furnished numerous switchable materials, some of them presenting hysteretic phase transitions ${ }^{6,7,10,11,12,16,20}$, and some of them presenting non-hysteretic phase transitions ${ }^{21,22}$. It is worth mentioning that the closely related family of dithiadiazolyl radicals has most recently furnished two examples of non-hysteretic spin transition compounds. ${ }^{23,24}$ Among the group of bistable DTA radicals, the case of 1,3,5-trithia2,4,6-triazapentalenyl (TTTA) is especially remarkable, since its bistability region spans 70 degrees and encompasses room temperature $\left(T_{\mathrm{c} \uparrow}=310 \mathrm{~K}, T_{\mathrm{c} \downarrow}=230\right.$ $\mathrm{K})^{7,25,26,27,28,29,30,31,32,33,34,35}$ (Figure 1a). The crystal structure of the 4-cyanobenzo-1,3,2dithiazolyl radical (4-NCBDTA, hereafter referred to as 1 , see Figure $1 \mathrm{~b}$ ), in turn, is a 
prototypical example of a DTA-based crystal featuring a non-hysteretic spin transition ${ }^{21}$. Its phase transition occurs at $T_{\mathrm{c}}=250 \mathrm{~K}$, a temperature in between the $T_{\mathrm{c} \uparrow}$ and $T_{\mathrm{c} \downarrow}$ values of TTTA. Last, it should be mentioned that in the DTA family there are also radicals, such as the $[1,2,5]$ thiadiazolo[3,4-f][1,3,2]benzodithiazol (TBDTA) radical ${ }^{12}$ (see Figure 1c), whose crystaline samples do not show any magnetic phase transition.

In previous works ${ }^{32,34,35}$, we have revealed key aspects of the physical and structural properties of TTTA as well as of the nature of its magnetic phase transition:

a) The different magnetic response of the LT and HT phases of TTTA originates in the different ways in which the TTTA radicals pile in each polymorph. The columns of radicals of $L T(T T T A)$ are distorted $\pi$-stacks that comprise $\pi$-dimers, i.e., pairs of nearly eclipsed radicals. Conversely, the columns of HT(TTTA) are regular m-stacks of radicals where each molecule exhibits a slipped overlap with its two adjacent molecules along the stacking direction. The dominant magnetic interactions in both polymorphs are found within the $\pi$-stacks. The very strong antiferromagnetic coupling between the spins of the two radicals forming the m-dimers of the LT(TTTA) phase explains the diamagnetism of this phase. The moderate antiferromagnetic coupling between adjacent radicals in the regular stacks of HT(TTTA), in turn, accounts for the reduced paramagnetism of this phase.

b) The dimerized stacks of LT(TTTA) correspond to a minimum energy structure in the potential energy surface (PES) of the system. In contrast, a full geometry optimization of the experimental crystal structure of HT(TTTA) led to an intrastack dimerization process. Yet, the resulting dimerized minimum structure is different from the structure of the LT polymorph because it features different interstack contacts.

c) The PES of a stack of TTTA radicals obeys a periodic double-well model (Figure 1d), where each of the wells represents one of the two equivalent dimerized configurations $(\cdots A-A \cdots A-A \cdots)_{n}$ and $(-A \cdots A-A \cdots A-)_{n}$ (in which $A-A$ represents an eclipsed pair and $A \cdots A$ represents the interaction between adjacent eclipsed pairs) and the transition state separating these two wells represents the regular structure $(\cdots A \cdots A \cdots A \cdots A \cdots)_{n}$. At low temperatures, the motion of the molecules within the columns is restricted to one of the two wells, while at higher temperatures, the molecules populate higher vibrational levels and eventually achieve enough energy to overcome the energy barrier. When this happens, the stacks explore both sides of the double well and continually hop from one well to the other one. This means that each TTTA radical continually exchanges (in the picosecond timescale) the adjacent neighbor (upper or lower) with which it forms a dimer. As a result of this particular dynamics, which was called Pair Exchange Dynamics (PED) ${ }^{34}$, the regular structure 
$(\cdots A \cdots A \cdots A \cdots A \cdots)_{n}$ becomes the most probable configuration (Figure $1 d$ ) and, thus, is the one observed by X-ray crystallography.

d) While the stacks of HT(TTTA) exhibit PED at $T>200 \mathrm{~K}$ (i.e, within the bistability region of TTTA), the stacks of LT(TTTA) remain dimerized over the whole range of temperatures up to at least $310 \mathrm{~K}$. The PED of the HT phase results in a large vibrational entropy, which stabilizes this polymorph (with respect to the LT polymorph) at finite temperatures. Therefore, the PED is a driving force of the LT $\rightarrow H T$ phase transition.

Despite the relevance of the above mentioned findings, there is one key aspect of the phase transition of TTTA that still remains poorly understood, namely, the origin of the hysteresis. In broader terms, the key factors controlling the existence or absence of hysteresis in the spin transitions of the different DTA-based switchable compounds are still largely unknown, thereby precluding a rational design of new DTA-based bistable materials. The work herein presented aims to shed light on this important issue by means of a combined computational and experimental work in which the properties and phase transition of a prototypical example of a DTA crystal undergoing a nonhysteretic spin transition are compared with those of a prototypical example of a bistable DTA crystal. Specifically, we will thoroughly analyze the magnetic response, the structural properties and the nature of the phase transition of 4-NCBDTA (1) and compare them with those of TTTA. This comparison, which is based on a computational study and new experiments designed to validate the theoretical predictions, will explain why TTTA is bistable while 1 is not and will bring to light the idea that the promotion of PED is a general mechanism to induce spin transitions in DTA-based crystals (and very likely in crystals of other organic radicals presenting $\pi-$ stacks), although this mechanism by itself does not suffice to generate bistability. 


\section{Results and Discussion}

The presentation of the results is organized as follows. We will first analyze the crystal packing of the two phases of 1 (Subsection 1). Second, we will investigate the microscopic origin of the different magnetic properties of the two phases of 1 (Subsection 2). Then, we will explore the potential energy surface of 1 and will establish whether or not its phases correspond to minimum energy structures (Subsection 3). After that, we shall examine the dynamics exhibited by the radicals in the two phases of 1 and establish the nature of its phase transition (Subsection 4). Following this, we will present experimental evidence based on magnetic and differential scanning calorimetry measurements that supports the computational results of the preceding subsections (Subsection 5). Finally, we will compare the properties and phase transitions of 4-NCBDTA and TTTA in order to understand why only the latter exhibits bistability (Subsection 6).

\section{Crystal packing analysis of the LT and HT polymorphs of 4-NCBDTA}

The analysis of the crystal packing of the LT and HT polymorphs of 1 was carried out using the previously-reported crystal structures refined at $180 \mathrm{~K}(1-180 \mathrm{~K}$ structure) and at $300 \mathrm{~K}\left(1-300 \mathrm{~K}\right.$ structure), respectively ${ }^{21}$. In both polymorphs, the radicals $(\mathrm{A})$ pile on top of each other giving rise to m-stacks. As in the TTTA case, the m-stacks of the LT polymorph are distorted or dimerized stacks that present an alternation between eclipsed and slipped pairs of radicals (Figure 2). If we denote the eclipsed pairs or $\pi-$ dimers by $A-A$, the dimerized stacks can be schematized as $(\cdots A-A \cdots A-A \cdots)_{n}$. Note that the dimerized stack can also be represented by the following isoenergetic configuration: (-A $\cdots A-A \cdots A-)_{n}$. The stacks of the HT polymorph of 1 , in turn, are regular m-stacks, where each molecule exhibits a slipped overlap with its two adjacent molecules along the stacking direction (Figure 2). This regular stacking motif, which can be schematized as $(\cdots A \cdots A \cdots A \cdots A \cdots)_{n}$, is analogous to that found in the HT phase of TTTA.

\section{Magnetic properties of the LT and HT polymorphs of 4-NCBDTA}

Given that the phase transition of 1 entails changes in the magnetic properties of the compound, it is important to identify the origin of these changes. In order to understand the different magnetic properties of 1 upon phase transition, we computed the value of 
the magnetic exchange coupling $(J)$ between pairs of radicals after excising them from the $1-180 \mathrm{~K}$ and $1-300 \mathrm{~K}$ crystal structures. According to our calculations (see Table 1 and Figure 3), the eclipsed pairs of $L T(1)$ and the slipped pairs of $H T(1)$ display strong antiferromagnetic couplings of ca. $-1700 \mathrm{~cm}^{-1}$ and ca. $-340 \mathrm{~cm}^{-1}$, respectively (similar to those computed for the two phases of $\operatorname{TTTA}^{32,35}$ ). These values are in agreement with the diamagnetic and weak paramagnetic response (reduced paramagnetism) observed, respectively, for the LT and HT phases of 1. On the other hand, it was found that the exchange couplings between radicals belonging to different stacks are much smaller than the couplings within the stacks (Table 1), thus showing that the dominant magnetic interactions in 1 occur along the m-stacks. Therefore, the change in the magnetic response of $\mathbf{1}$ upon phase transition can be traced to the structural changes in the m-stacks, a scenario that is completely analogous to that found in the TTTA case.

\section{Optimum structure of the LT and HT polymorphs of 4-NCBDTA}

Variable-cell geometry optimizations of $\mathbf{1}$ starting from the atomic coordinates and lattice parameters of the previously reported $1-180 \mathrm{~K}$ and $1-300 \mathrm{~K}$ crystal structures were carried out in order to establish whether the LT and HT polymorphs are minimum energy structures in the potential energy surface of the crystal. The model system employed in our calculations, which was designed to represent the LT and HT crystals on an equal footing, was a supercell containing sixteen 4-NCBDTA radicals, arranged in 4 columns of 4 molecules each (see Supporting Section S1).

The geometry optimizations of both the LT and HT polymorphs ended in the same optimized structure, which we label as $1-0 \mathrm{~K}$, since the minimum-energy structures can be understood as the 0-K crystal structure. As shown in Figure 4 (see also Table S1), $1-0 \mathrm{~K}$ preserves the eclipsed $\pi$-dimers and is in excellent agreement with the 1-180K crystal structure. The fact that the optimization starting from $1-300 \mathrm{~K}$ resulted in an intrastack dimerization process that produced the same 1-0K structure demonstrates that the regular stacking motif observed for the HT phase does not correspond to a minimum in the PES of the system. Although the HT phase of TTTA was also found not to be a minimum in the PES ${ }^{34}$ there is a crucial difference between TTTA and 1. In the former, each phase (LT and HT) has a different optimized minimum-energy structure (the two phases differ in the relative orientation of the TTTA radicals and in the interstack contacts), whereas the two phases of 1 have the same optimized minimumenergy structure. 


\section{Dynamics of the LT and HT polymorphs of 4-NCBDTA}

To assess the importance of vibrational entropy in the structures of 4-NCBDTA (1), we conducted ab initio molecular dynamics (AIMD) simulations at 180, 240, 260 and 300 $\mathrm{K}$, in the canonical ensemble using the Car-Parrinello propagation scheme. ${ }^{36}$ It is worth mentioning that this computational strategy has been successfully used in the past to characterize the magnetic and structural properties of other organic radicals ${ }^{35,37}$.

The average structure obtained from the AIMD simulations at $300 \mathrm{~K}$ is very close to the regular structure observed by X-ray crystallography at this temperature (Figure $2 a$ and Figure $4 \mathrm{~d}$ ) including the uniform separation of the molecules of 1 . The good agreement between the computed and experimental structures, together with the good agreement between the computed and experimental thermal ellipsoids (see Supporting Section S.2.1), validates the choice of the model system and the AIMD simulations performed to investigate the dynamical properties of 1 . The regular stacks obtained when considering the thermal fluctuations suggest that PED is operative at this temperature. The time-resolved evolution of the centroid-centroid distance $\left(d_{\mathrm{CM}}\right)$ between neighboring molecules (shown in Figure 5) confirms that this is indeed the case. Such time-resolved evolution shows that each pair of molecules presents roughly the same kind of intermolecular vibrations, in terms of their amplitude (ca. $1 \AA$ ) and of their mean value (ca. $3.68 \AA$ ), which indicates that the three pairs of the stack are equivalent. Our results thus show that the regular stacks observed in the 1-300K crystal structure are the average structures resulting from an intrastack $(\cdots A-A \cdots A-A \cdots)_{n} \leftrightarrow(-A \cdots A-A \cdots A-)_{n}$ dynamics whereby eclipsed $\pi$-dimers between radicals are continually formed and broken on the picosecond timescale. Compound 1 is the second example of a DTA radical (the first one was TTTA) whose HT phase has been shown to exhibit PED. This strongly suggests that such PED is a general phenomenon within the family of switchable DTAs. It should also be mentioned that the timescale in which the PED occurs in the HT phase of 1 at $300 \mathrm{~K}$ coincides with the timescale associated with the PED in the HT polymorph of TTTA at the same temperature.

At $180 \mathrm{~K}$, the average structure obtained from the AIMD simulations, which is in excellent agreement with the $1-180 \mathrm{~K}$ crystal structure, displays the common alternation of eclipsed and slipped pairs, the former type having shorter inter-radical N-N distances than the latter (Figure $2 \mathrm{~b}$ and Figure 4c). As shown in Figure 5, the intermolecular vibrations of the eclipsed pairs can easily be distinguished from those of the offset pair 
since the corresponding $d_{\mathrm{CM}}$ values oscillate around a different average value. Therefore, the eclipsed $\pi$-dimers are preserved over the whole AIMD trajectory, that is to say, the PED is inactive at this temperature. The good agreement between the computed and experimental thermal ellipsoids (see Supporting Section S2.1) fully supports our results. The average structure of the stacks obtained from the AIMD simulations run at 240 and $260 \mathrm{~K}$ (i.e., at temperatures slightly below and slightly above the previously reported spin-transition temperature ${ }^{21}$ ) is different depending on the stack of molecules that we analyze. Two columns display a totally dimerized configuration similar to that of $1-0 \mathrm{~K}$, whereas the other two display a nearly-regular arrangement (see Supporting Section S2.2), which indicates that some PED events have occurred along the simulations. This also suggests that the change in the dynamics of one stack upon heating/cooling occurs independently of the adjacent stacks.

The presence of PED processes in 1 can be further analyzed on the basis of the 2dimensional pair-distribution functions (2D-PDF) associated with the interplanar distance $\left(d_{\mathrm{ip}}\right)$ and the relative slippage $\left(d_{\mathrm{sl}}\right)$ (see Figure 6 and also Supporting Section S2.3) These variables decompose the centroid-centroid distance $\left(d_{\mathrm{CM}}\right)$ between neighboring molecules, and allow for a precise analysis of the thermal fluctuations undergone by 1 (see Section S3.2 for an extended analysis). The 2D-PDF of our AIMD simulation at $180 \mathrm{~K}$ presents two peaks. The left-hand side peak corresponds to the eclipsed pair, and the right-hand side one corresponds to the slipped pair. The presence of these two well-defined peaks is fully consistent with the absence of PED at this temperature (Figure $5 \mathrm{a}$ ) and the dimerized average structure obtained from the AIMD simulations at $180 \mathrm{~K}$. As the temperature is increased to 240 and $260 \mathrm{~K}$, the two peaks broaden and slightly overlap, which is the result of larger-amplitude oscillations and some pair-exchange events. Finally, at $300 \mathrm{~K}$ the PED is fully operative (Figure 5b) and, as a result, the two peaks completely merge into a single broad peak, which indicates that the most-probable structure is the regular stack of radicals (Figure $4 \mathrm{~d}$ ). The evolution of the 2D-PDFs displayed in Figure 6, which is similar to that observed for the monoclinic polymorph of TTTA ${ }^{34}$, reveals that the activation of the PED is a gradual process that entails an order-disorder transition within the m-stacks.

Both the order-disorder transition of 4-NCBDTA crystal and the results obtained upon optimization of its two polymorphs can be rationalized on the basis of the energy profile displayed in Figure 7, which shows the variation of the potential energy of an isolated $\pi$-stack of 4-NCBDTA radicals (considering periodic boundary conditions along the 
stacking direction) as an intrastack dimerization process takes place. The shape of this profile is that of a double-well model potential, where the two minima correspond to two degenerate dimerized configurations and the maximum to the regular structure observed at $1-300 \mathrm{~K}$ crystal structure. This profile demonstrates that the regular stacking motif is not a minimum energy structure in the PES of the system. It also shows that the PED arises from a dynamic interconversion between two degenerate dimerized stacks that takes place when the system has enough thermal energy to overcome the barrier separating these two dimerized stacks.

The gradual activation of the PED process observed for 1 strongly suggests that the phase transition undergone by this material is a second-order phase transition (i.e, a gradual transition without an abrupt release of thermal energy and without significant thermal hysteresis). However, this conclusion is in contradiction with the abrupt variation observed in the magnetic susceptibility curve of 1 at $250 \mathrm{~K}$ in Ref. 21 , which suggests that the phase transition of this crystal should be first-order. In order to address this contradiction, the temperature-dependence of the magnetic susceptibility of 1 was re-measured under thermal equilibration conditions and differential scanning calorimetry (DSC) experiments were conducted. The results of these new experiments will be presented in the next subsection.

\section{Experimental confirmation of the theoretical predictions of the phase transition of 4-NCBDTA}

As mentioned above, the final step of our study was aimed at checking the validity of the most relevant theoretical conclusions drawn for compound 1. For such a task, a sample of 4-NCBDTA was synthesized and crystallized, using procedures slightly modified from those reported in the literature ${ }^{21}$ (see Supporting Section S3). X-ray powder diffraction experiments at 180 and $300 \mathrm{~K}$ showed that the high and low temperature phases are in good agreement with the previously reported unit cells and structures (see Supporting Section S4). In fact, single-crystal diffraction analysis (see Supporting Section S5) confirms that the high- and low-temperature phases of 4NCBDTA obtained in this work correspond to the previously reported structures ${ }^{21}$. In addition, EPR and HRMS data are also consistent with the expected molecular formula and nitrogen-centered radical (see Supporting Section S3 and Supporting Section S6).

The magnetic susceptibility of a polycrystalline sample of 1 was collected every $5 \mathrm{~K}$ in the $225-305 \mathrm{~K}$ range by cooling from room temperature and then warming the sample 
back to room temperature (Figure 8a and Supporting Section S7). Special care was payed to make sure that thermal equilibration was achieved at each temperature before collecting the value of the magnetic susceptibility. In addition, the sample was recentered before each data point was collected through the region of the transition. The data thus obtained (Figure 8a) clearly shows a gradual, non-abrupt phase transition in the 260-272 $\mathrm{K}$ temperature range with minimal, if any, hysteresis. As expected, the magnetic susceptibility value becomes negative as the LT material becomes fully diamagnetic. Both the gradual nature of the transition and the temperature range in which it occurs are in good agreement with the predictions of the 2D-PDF analyses discussed above, thus supporting our conclusion that the phase transition of $\mathbf{1}$ is second order.

In order to obtain an independent evaluation of the first or second order nature of the phase transition of 1, DSC experiments were also performed over the 240 to $305 \mathrm{~K}$ range. The DSC curves were obtained at three cooling/heating rates. As shown in Figure 8b (see also Supporting Section S8), in all three DSC curves the phase transition was observed in the $260-272 \mathrm{~K}$ range, which is fully consistent with the temperature range observed in the new magnetic susceptibility measurements. Unlike a first-order phase transition (which would be expected to produce an abrupt release/absorbance of thermal energy at the transition temperature), a smooth release of thermal energy is observed, thus providing an independent experimental confirmation that the magnetic phase transition of 1 is second order.

To conclude this subsection, let us mention that the phase transition of 4-NCBDTA can be classified as a spin-Peierls transition because it involves the transformation of regular $S=1 / 2$ antiferromagnetic chains into dimerized stacks presenting an alternating exchange coupling between adjacent spins. However, the phase transition of this material features some unique characteristics (the absence of a minimum energy structure in the PES associated with the regular stacking motif, the presence of a PED process in the high-temperature phase and the fact that it is an order-disorder transition) that are nor shared by all spin-Peierls-type transitions. For this reason, the use of the PED and order-disorder concepts allows for a better description of the nature of the phase transition of 4-NCBDTA. 


\section{Rationalizing the different nature of the phase transitions of TTTA and 4- NCBDTA}

The results so far presented provide solid evidence that the non-hysteretic spin transition of 4-NCBDTA is a second order phase transition. Such a second-order phase transition is in contrast with TTTA, whose hysteretic spin transition is first-order. In order to understand why the nature of the phase transition of these two compounds is different, let us first recapitulate their main common characteristics:

a) The LT polymorphs of both materials present dimerized m-stacks, while the corresponding HT polymorphs exhibit regular m-stacks.

b) The LT polymorphs of both materials present very strong antiferromagnetic interactions within the $\pi$-stacks, which are responsible for the diamagnetic character of these crystals. Conversely, in the corresponding HT polymorphs, the intrastack exchange interactions become much weaker and are responsible for the dominant, but weak, paramagnetic character of these two crystals.

c) The HT polymorphs of the two compounds are not minimum energy structures in the PES of the crystal. The regular stacking motifs of these polymorphs are the average structures resulting from PED processes, which are operative above the spin-transition temperatures. The PED mechanism is the source of a large vibrational entropy that stabilizes the HT polymorphs above the spin-transition temperature.

The common characteristics between 4-NCBDTA and TTTA provide evidence that in DTA-based materials the thermal promotion of an intrastack PED process constitutes a general mechanism to trigger both hysteretic and non-hysteretic spin transitions. However, the existence of a PED process in a DTA compound is not sufficient to determine whether a spin transition occurs with or without hysteresis.

In order to identify what controls the presence or absence of bistability in a spin transition of a DTA crystal, we need to focus on the differences between 4-NCBDTA and TTTA. The key difference between these two materials is that the former requires only a single, intrastack stuctural transition (via PED), while the latter requires the same intrastack change plus the addition of an interstack rearrangement (Figure 9). The optimized, dimerized minimum potential energy structure of 4-NCBDTA at $0 \mathrm{~K}$, regardless of whether the optimization starts from the experimentally observed HT or LT phase, corresponds to the experimentally observed LT phase. In the optimization beginning from the observed, regular, HT phase, a barrierless, intrastack dimerization process is observed. This requires only a change in the intrastack intermolecular 
distances and does not require a change in the interstack interactions; it is a fully reversible process that leads to two degenerate structures. It is critical to note that the potential energy surface relevant to the phase transition exhibits only two energetically and topologically equivalent miniumum energy structures. Therefore, the spin transition observed in 4-NCBDTA can be described based upon a temperature dependent change in the free energy surface, resulting from an order/disorder transition as shown in Figure 1d.

Conversely, in TTTA the scenario is more complex because it requires two distinct types of structural transformations: an intrastack and an interstack (Figure 9a) transformation. The optimized, dimerized structure of TTTA, beginning from the observed LT, triclinic packing structure, corresponds to that experimentally observed LT phase. In contrast, as shown in Ref. 34, the optimized, dimerized structure beginning from the experimentally observed, regular, HT monoclinic phase, does not correspond to the observed LT structure, nor to any experimentally observed phase of TTTA. During the optimization beginning from the HT phase, a barrierless, intrastack dimerization process is observed which again requires only a change in the intermolecular, intrastack distances, exactly as was observed in the case of 4NCBDTA. Since this optimized, dimerized structure shows distinct interstack interactions compared to the experimentally observed LT phase, we can conclude that the interstack rearrangement upon phase transition of TTTA necessarily implies the presence of an energetic barrier, which is the origin of the observed bistability. Therefore, the critical observation in TTTA is the requirement for both the intrastack transformation as observed previously in 4-NCBDTA and a non-degenerate interstack rearrangment (Figure 9a). which connects two distinct crystal structure minima and is responsible for the hysteresis and the first-order character of its phase transition. 


\section{Conclusions}

In the study herein presented, which is based on a joint effort between theory and experiment, we have carried out an exhaustive analysis of the phase transitions of prototypical examples of DTA radicals undergoing both hysteretic and non-hysteretic spin transitions between LT diamagnetic and HT paramagnetic phases. The mechanism of Pair Exchange Dynamics (PED), that is, the averaging of structures arising from a dynamic interconversion between two equivalent dimerized configurations, illustrated as $(\cdots A-A \cdots A-A \cdots)_{n} \leftrightarrow(-A \cdots A-A \cdots A-)_{n}$, is operative in the high temperature phases of these systems resulting in uniform spacing within the radical stacks. At high temperatures, these uniform stacks constitute a minimum in the free energy of the system as a result of entropy contributions, although they are not a minimum in the potential energy surface. The theoretical results herein reported predict that the phase transition that connects the HT and LT phases of 4-NCBDTA is gradual and second order rather than abrupt and first order. Experimental results using DSC and magnetic susceptibility analyses show that compound 1 does indeed undergo a non-abrupt second-order phase transition as a result of the onset of a PED process. The combination of the theoretical and experimental results demonstrates that the entire transition can be described by a single potential energy surface.

The second-order transition of 4-NCBDTA is in contrast to what is observed for TTTA, whose phase transition exhibits a large and abrupt heat change that is characteristic of a first-order transition ${ }^{26}$. In TTTA, the onset of an intrastack PED process is accompanied by an interstack structural rearrangement resulting in a system which crosses between two distinct minima with an associated energy barrier. This is responsible for the observed hysteresis and accounts for the difference between the behaviours of 4-NCBDTA and TTTA.

The fact that both compounds present the same kind of intra-stack thermal vibrations (ie. PED) suggests that the PED process is a common feature in the DTA family of radicals. The PED process will likely prove to be a key concept to rationalize not only the properties of other DTA-based materials undergoing hysteretic spin transitions but also the gradual variation of the magnetic susceptibility of other DTA radicals featuring $\pi$-stacks (e.g. the quinoxaline-1,3,2-dithiazolyl radical ${ }^{38}$ and the $\alpha$ polymorph of the pyridyl-1,3,2-dithiazolyl radical ${ }^{39}$ ). Overall, it becomes now clear that the initiation of a PED process and the concomitant gradual second-order phase transition is sufficient to 
endow DTA organic radicals with spin-switching properties. Notwithstanding the great potential of the activation of a PED process as an effective tool to design new switchable materials, it should be taken into account that this type of transition, that occurs in isolated regions of the structure (i.e. within the stacks), does not suffice to generate a region of bistability. The opening of a hysteresis loop calls for the presence of two distinct energy minima and a barrier to the interconversion between them which is required for the presence of bistability in the DTA family.

The potential application of these studies to the rational design and analysis of such molecules, and switchable magnetic materials in general, is clear. Future studies should explore how the onset of a PED process can be tuned by means of intermolecular interactions (both intra- and inter-stack) to control the nature and temperature of the spin transition. One of the main factors governing the onset of the PED is the strength of the long, multicenter bond (alternatively called pancake bond ${ }^{40}$ ) between DTA radicals in the $\pi$-dimers. In this respect it should be mentioned that the large impact of small geometrical changes on the strength of long, multicenter bonds between planar organic radicals ${ }^{24,41,42}$ bodes well for the design of new switchable DTA-based materials with a large variety of spin-transition temperatures. In addition, investigations to determine how broadly applicable the initiation of a PED process may be in related materials featuring stacks of planar organic radicals are in progress. 


\section{Methods}

The description of the methodology employed in this article is organized as follows. We will first describe the computational methodology employed to obtain the results presented in subsections 3.2, 3.3 and 3.4 of the Results and Discussion. After that, we will detail the methodology employed in the experimental part of the work.

\section{Computational Details}

i) Evaluation of the magnetic exchange couplings (subsection 3.2 of Results and Discussion)

The $J$ values of the radical pairs of the X-ray crystal structures were computed at the UB3LYP $\mathrm{P}^{43} / 6-31+\mathrm{G}(\mathrm{d})^{44}$ level as implemented in Gaussian $09^{45}$ by using the isotropic Heisenberg Hamiltonian, $\widehat{H}=-2 \sum_{A, B}^{N} J_{A B} \cdot \hat{S}_{A} \cdot \hat{S}_{B}$. The Broken-Symmetry (BS) approach has been used to properly describe the open-shell low-spin (singlet) state $\left(U_{A B}=E^{B S, S}-E^{T}\right)$.

ii) Optimum structure of the $L T$ and $H T$ polymorphs of 4-NCBDTA (subsection 3.3 of Results and Discussion)

Plane wave pseudopotential calculations were employed for the variable-cell geometry optimizations of $\mathbf{1}$. These calculations, in which the atomic positions and the lattice parameters were optimized simultaneously, were carried out using the PBE exchangecorrelation functional ${ }^{46}$ within the spin unrestricted formalism, together with Vanderbilt ultrasoft pseudopotentials ${ }^{47}$ and gamma-point sampling of the Brillouin zone as implemented in the QUANTUM ESPRESSO package ${ }^{48}$. In these calculations, the semiempirical dispersion potential introduced by Grimme ${ }^{49}$ was added to the conventional Kohn-Sham DFT energy in order to properly describe the van der Waals interactions between the different 4-NCBDTA molecules. The parametrization employed in our work is the so-called DFT-D2 parametrization. It is noted that a series of recent benchmark calculations have shown that the use of PBE together with the Grimme furnishes good predictions for the structure and cohesive energies of molecular crystals. ${ }^{50}$ Indeed, the PBE-D2 scheme has already been shown to provide a difference in cohesive energies between the two polymorphs of TTTA that is in good accordance with the experimental data. ${ }^{35}$ The plane wave basis set was expanded at a 
kinetic energy cutoff of $70 \mathrm{Ry}$. The number of plane waves was kept constant throughout the variable-cell relaxations. A constant number of plane waves implies no Pulay stress but a decreasing precision of the calculation as the volume of the supercell increases. The large cutoff employed in these calculations ensures that the artefacts arising from this change of precision are negligible.

iii) Dynamics of the $L T$ and HT polymorphs of 4-NCBDTA (subsection 3.4 of Results and Discussion)

The Car-Parrinello molecular dynamics simulations of 4-NCBDTA were carried out with the CPMD package ${ }^{51}$. These calculations were also done using the PBE exchangecorrelation functional ${ }^{46}$ within the spin unrestricted formalism, together with Vanderbilt ultrasoft pseudopotentials ${ }^{47}$ and gamma-point sampling of the Brillouin zone. In these simulations, the plane wave basis set was expanded at a kinetic energy cutoff of 25 Ry. The molecular dynamics time step was set to 4 a.u. and the fictitious mass for the orbitals was chosen to be 400 a.u. All dynamic simulations were performed in the canonical ensemble using Nose-Hoover chain of thermostats ${ }^{52}$ in order to control the kinetic energy of the nuclei and the fictitious kinetic energy of the orbitals.

The potential energy profile displayed in Figure 7 has been computed with CPMD using the same setup as that employed in the molecular dynamics simulations.

\section{Experimental methodology}

\section{i) Magnetic Susceptibility Data}

Magnetic susceptibility data were measured using a freshly sublimed batch of radical 1 on a Quantum Design MPMS-XL SQUID magnetometer. A sample of 1 was packed into a \#3 gelatin capsule and mounted in the SQUID. Susceptibility data were taken over the temperature range from $290 \mathrm{~K}$ to $249 \mathrm{~K}$ and then back to $274 \mathrm{~K}$ in an applied field of 5000 Oe. To ensure accuracy of the data, the sample's temperature was moved towards each target temperature at a slow rate of $2 \mathrm{~K} / \mathrm{min}$ and then allowed equilibrate until it had stabilized within $+/-0.2 \mathrm{~K}$ of the target temperature before each data point was collected. The data were corrected for the background signal of the sample holder, and for the diamagnetic contributions of the constituent atoms (estimated using Pascal's constants, which were taken from Ref. 53). 


\section{ii) Differential Scanning Calorimetry}

DSC analysis of radical 1 was performed with freshly sublimed material with careful exclusion of moisture. Data was collected on a Perkin Elmer DSC 7 with Pyris 9.0.2.0193 software.

\section{Supporting Information}

Crystal structure data in CIF format for the LT and HT phases of 4-NCBDTA (these structures have been deposited in the CCDC with deposition numbers 1524306 and 1524307). The Supporting Information also includes: detailed information on the model system employed in the computational studies, an extended analysis of the AIMD trajectories, the synthetic procedure used to obtain 4-NCBDTA, analysis of the powder diffraction X-ray data, an analysis of the single-crystal X-ray data, EPR data, magnetic susceptibility data and differential scanning calorimetry data.

\section{Acknowledgements}

We acknowledge the Spanish Government for financial support (Projects MAT201125972 and MAT2014-54025-P) and BSC and CSUC for the allocation of massive computer time. S.V. is thankful to the LabEx-Chemistry of Complex Systems for a postdoctoral grant (ANR-10-LABX-0026CSC). Funding for the experimental portions of this work was provided by Clark University's faculty startup funds for CEJ. We would also like to thank Professor Fred Greenaway for assistance with electron paramagnetic resonance data collection, and Peter Müeller for assistance with powder diffraction Xray data collection. Finally, we are thankful to the Catalan DURSI (Grant 2014SGR1422). 
a)<smiles>n1snc2c1SNS2</smiles>

Low Temp. (LT)

Diamagnetic

Crystal

b)<smiles>CN1Sc2cccc(C#N)c2S1</smiles>

Low Temp. (LT) Diamagnetic Crystal

$$
\begin{gathered}
\begin{array}{c}
\text { abrupt change } \\
\text { at } 310 \mathrm{~K}
\end{array} \\
\begin{array}{c}
\text { High Temp. (HT) } \\
\text { Paramagnetic } \\
\text { at } 230 \mathrm{~K}
\end{array}
\end{gathered}
$$

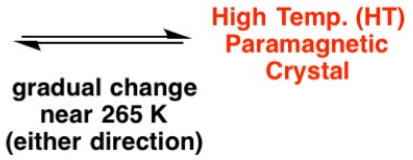

c)

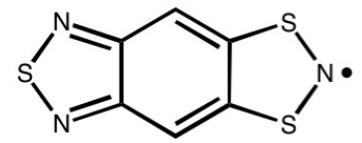

no spin transition

d)

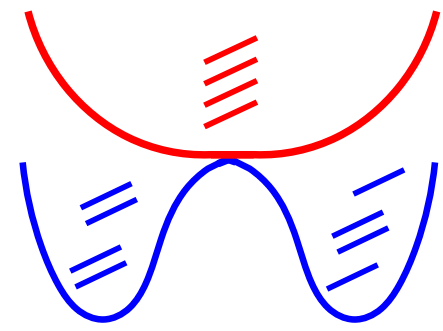

Single free-energy minimum above critical temperature due to the PED

Double free-energy minima below critical temperature

Figure 1. (a) Molecular structure of the TTTA radical and scheme of the main characteristics of its phase transition. (b) Molecular structure of the 4-NCBDTA radical and scheme of the main characteristics of its phase transition. (c) Molecular structure of the TBDTA radical, which does not undergo any spin transition. (d) Scheme showing the temperature dependence of the free energy profile of one column of TTTA radicals with respect to an intrastack dimerization process. 
(a) $1-300 \mathrm{~K}$
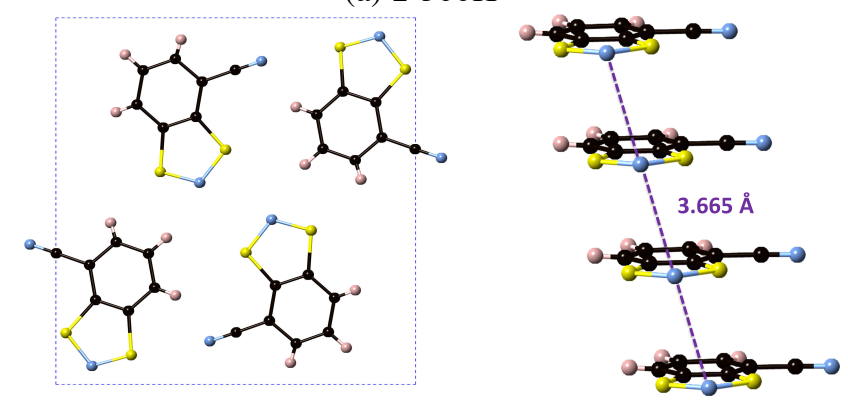

(b) $1-180 \mathrm{~K}$
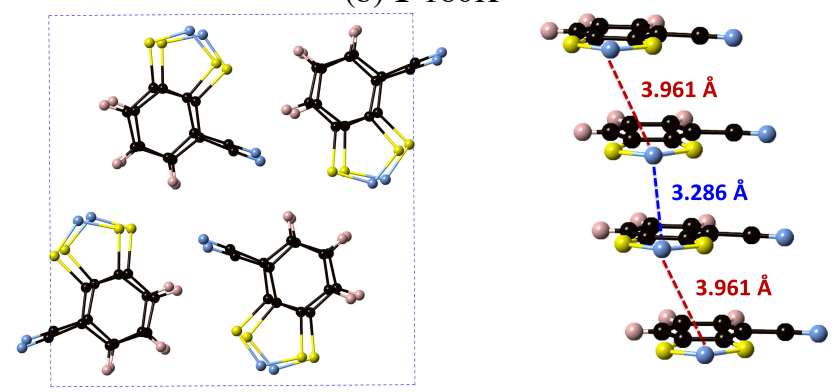

Figure 2. Top- and side-views of the experimental structure of the HT (a) and LT $(b)$ polymorphs of 1 . The X-rays structure of HT was determined at $300 \mathrm{~K}$, while that for LT was obtained at $180 \mathrm{~K}$. The distances displayed in the Figure correspond to the distances between the $\mathrm{N}$ atoms of the S-N-S moieties of adjacent radicals. 

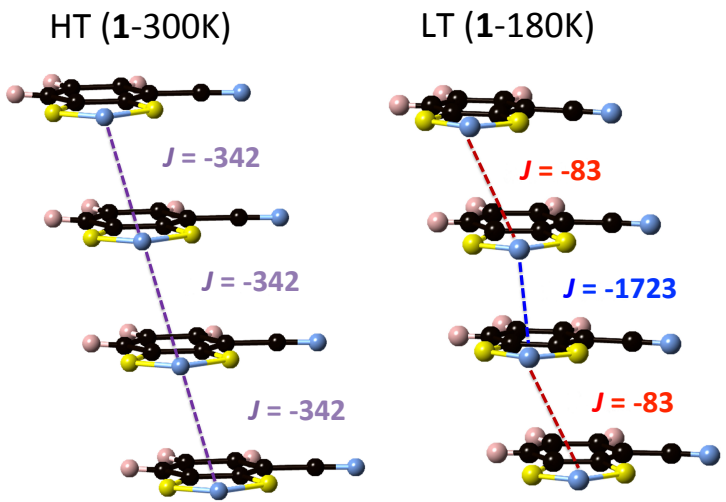

Figure 3. Magnetic exchange couplings along the m-stacks of the LT and HT polymorphs of 1. All the values of $J$ are given in $\mathrm{cm}^{-1}$. 
a)

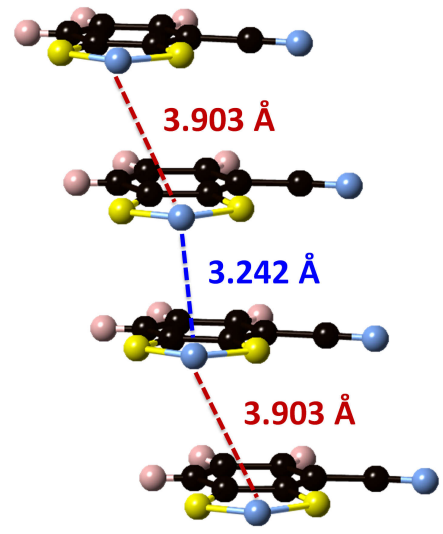

c)

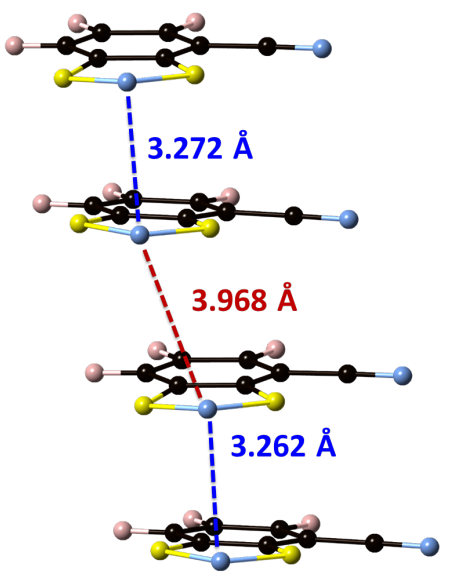

b)

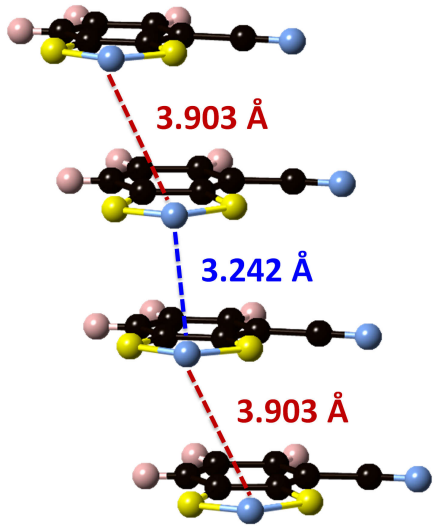

d)

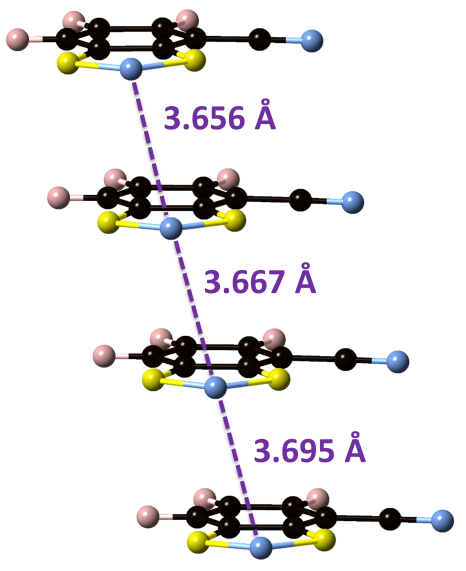

Figure 4. Structure of a stack of 1-0K after the variable-cell geometry optimization starting from the $1-180 \mathrm{~K}$ structure (a) and starting from the $1-300 \mathrm{~K}$ structure (b). Average structure of a stack along the AIMD trajectory at $180 \mathrm{~K}$ (c) and $300 \mathrm{~K}$ (d). The distances displayed in the Figure correspond to the distances between the $\mathrm{N}$ atoms of the S-N-S moieties of adjacent radicals. In the dimerized structures shown in (a), (b) and (c), the N-N distances marked in blue and red correspond to the eclipsed and slipped pairs, respectively. 
(a) $180 \mathrm{~K}$
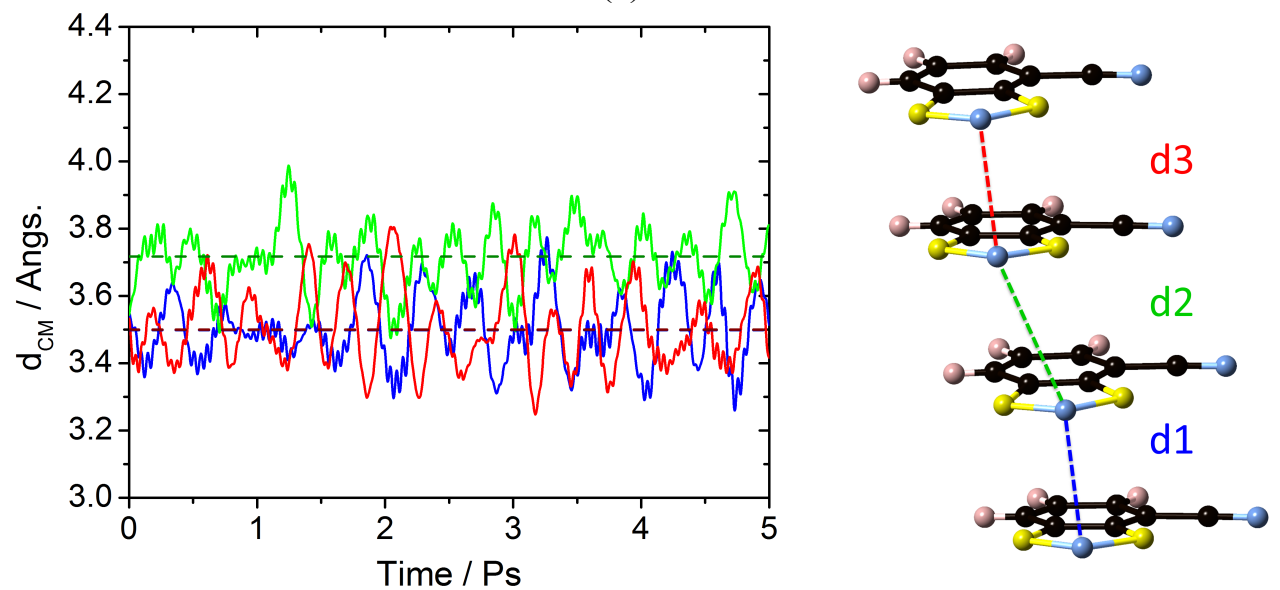

(b) $300 \mathrm{~K}$
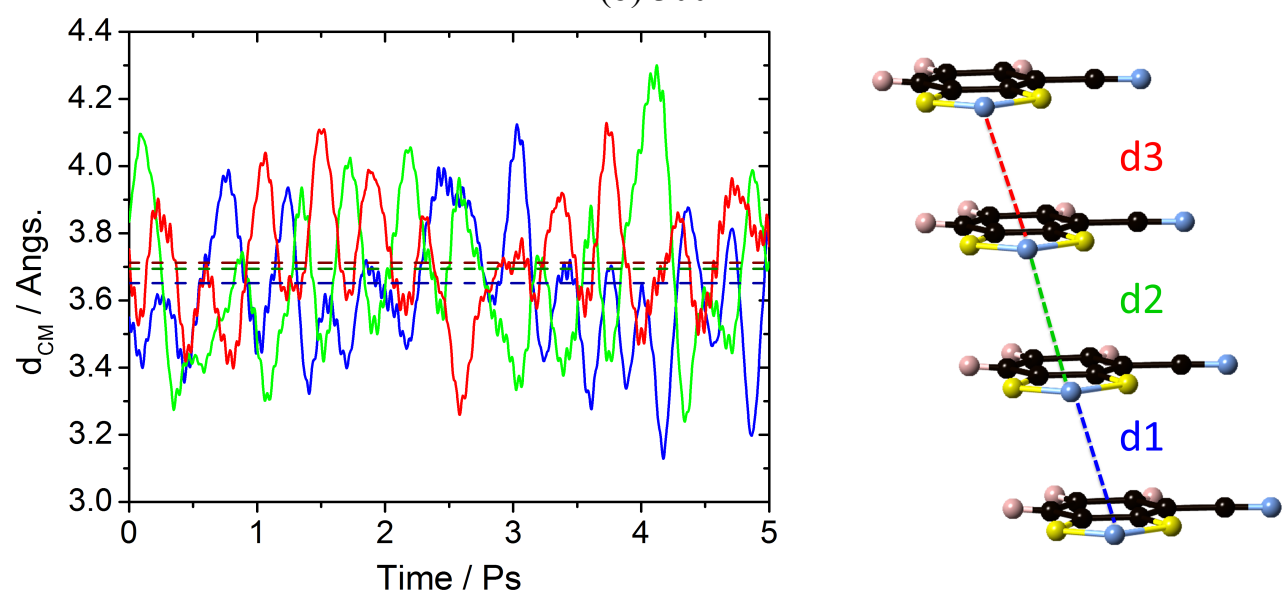

Figure 5. Time-resolved evolution of the centroid-centroid distance $\left(d_{\mathrm{CM}}\right)$ between adjacent molecules of 1 along the AIMD-trajectories at (a) 180 and (b) $300 \mathrm{~K}$. 


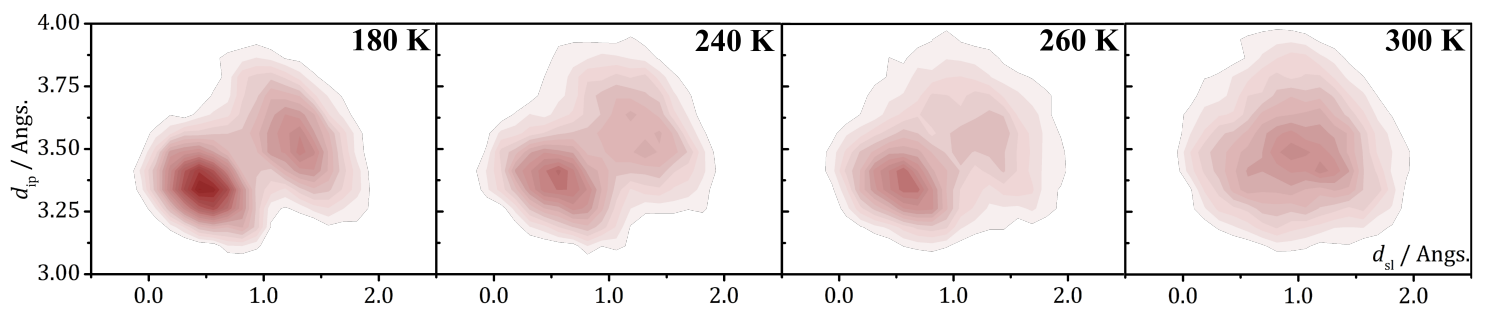

Figure 6. 2D-PDF's associated with the interplanar distance $\left(d_{\mathrm{ip}}\right)$ and the relative slippage $\left(d_{\mathrm{sl}}\right)$ between adjacent molecules of 1 along the AIMD-trajectories at $180 \mathrm{~K}, 240 \mathrm{~K}, 260 \mathrm{~K}$ and $300 \mathrm{~K}$ (from left to right) 


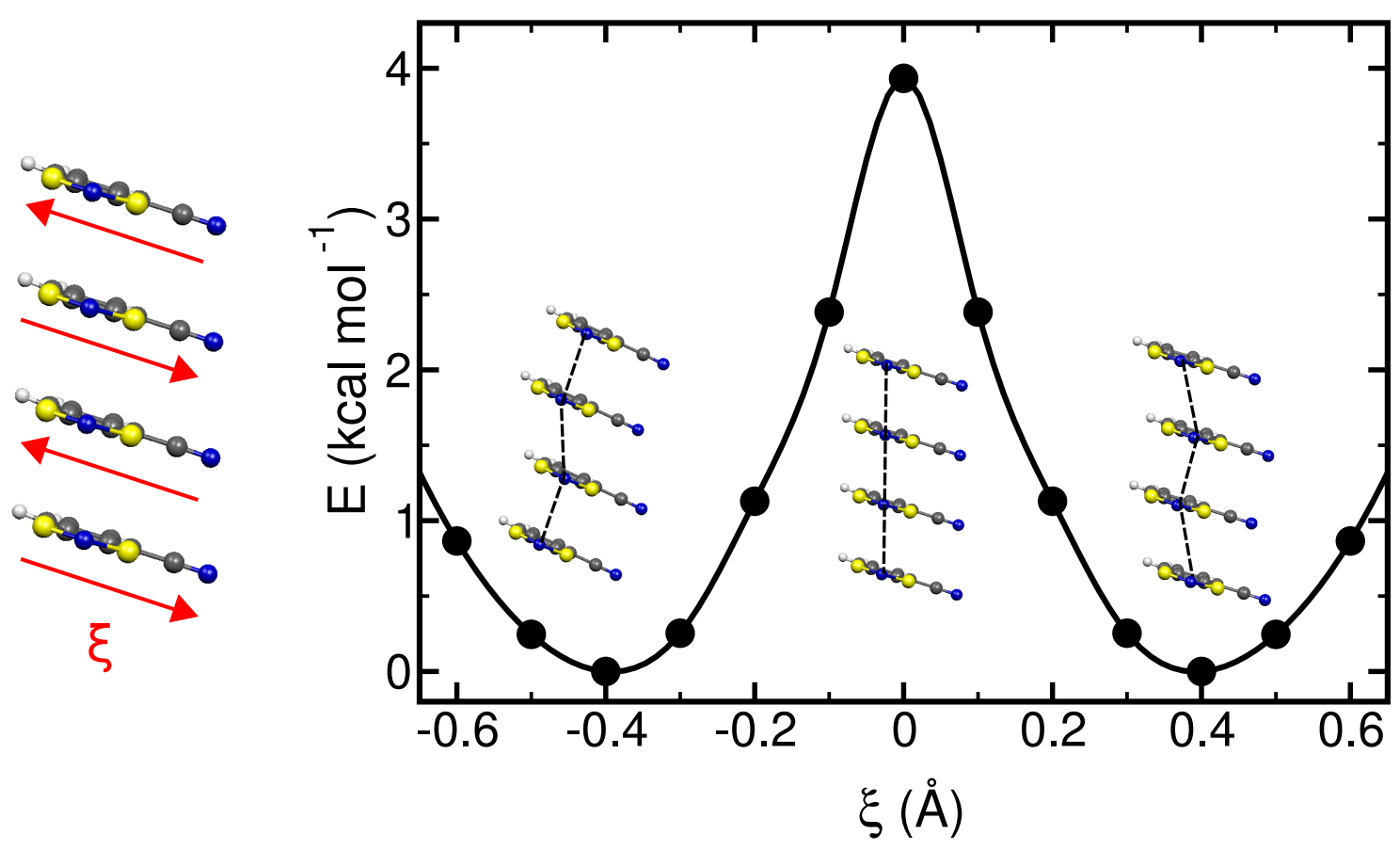

Figure 7. Potential energy profile of an isolated stack of 4-NCBDTA radicals along the reaction coordinate $\xi$. This reaction coordinate, which is defined in the left-most scheme, describes a sliding motion of the radicals that preserves the interplanar distance between them. The profile has been computed with periodic boundary conditions along the stacking direction. The regular structure shown at $\xi=0$ corresponds to the $1-300 \mathrm{~K}$ crystal structure. 
A. Magnetic Susceptibility of Radical 1 in a 5 kOe Field

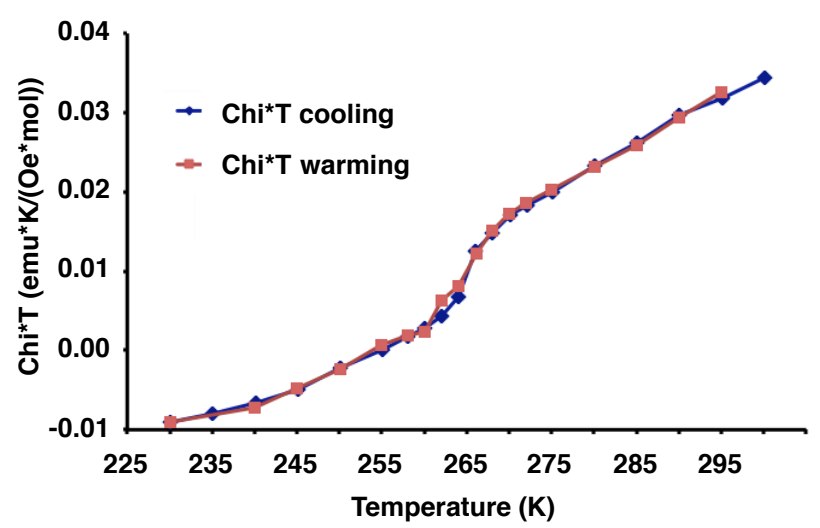

B. Differential Scanning Calorimetry of Radical 1

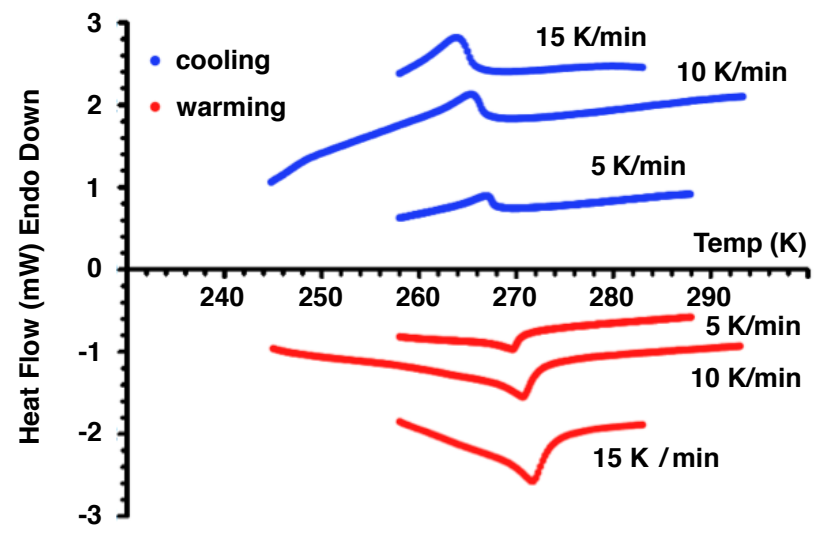

Figure 8. Experimental analysis of radical 1's phase transition. (a) Molar magnetic susceptibility data and (b) differential scanning calorimetry data at different heating and cooling rates. The small differences observed between the cooling and heating curves are within normal experimental error when measuring magnetization values near zero. In the raw data, the magnetization transitions from positive to negative at $265 \mathrm{~K}$. 
a)

TTTA

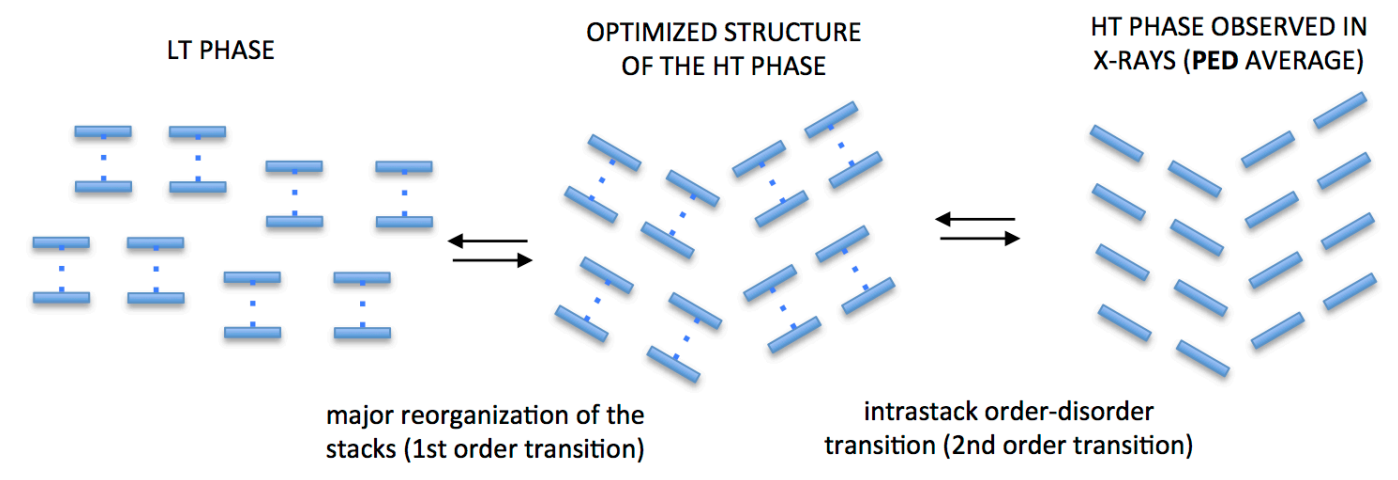

b)

4-NCBDTA

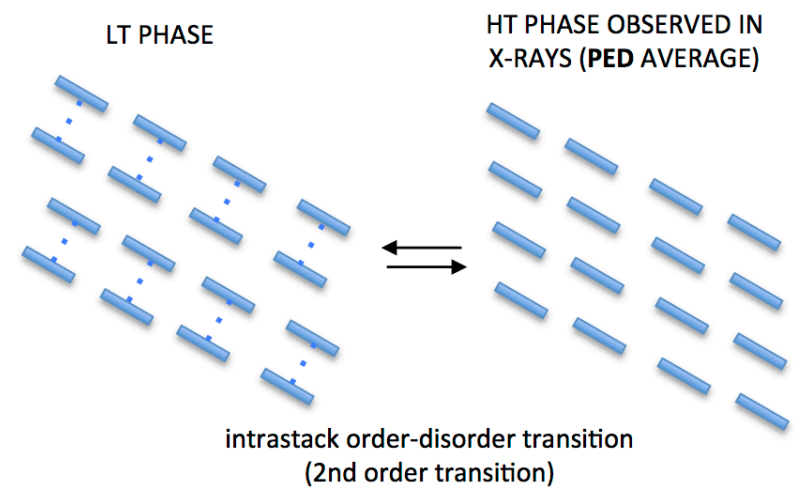

Figure 9. Cartoon depicting the differences between the phase transition of TTTA (a) and 4NCBDTA (b). While the phase transition of 4-NCBDTA involves only an intrastack orderdisorder transition, the phase transition of TTTA involves both an intrastack order-disorder transition and an interstack transformation. 
Table 1. Magnetic exchange couplings $(J)$ between selected radical pairs, extracted from the experimentally reported $1-180-\mathrm{K}$ and $1-300-\mathrm{K}$ crystal structures. Pairs can be identified using the $\mathrm{N}-\mathrm{N}$ distance between the $\mathrm{N}$ atoms of their S-N-S moieties.

\begin{tabular}{cccc} 
& $\mathbf{1 - 1 8 0 \mathrm { K }}$ & & \multicolumn{1}{c}{$\mathbf{1 - 3 0 0 \mathrm { K }}$} \\
$d(\mathrm{~N}-\mathrm{N})^{\mathrm{a}} / \AA$ & $J / \mathrm{cm}^{-1}$ & $\mathrm{~d}(\mathrm{~N}-\mathrm{N}) / \AA$ & $J / \mathrm{cm}^{-1}$ \\
3.286 & -1723.1 & 3.665 & -341.9 \\
3.961 & -82.57 & 3.284 (Interstack $^{\mathrm{b}}$ & -5.0
\end{tabular}

a The different pairs of radicals have been identified using the distance between the $\mathrm{N}$ atoms of their S-N-S moieties, since this $\mathrm{N}$ atom is the atom that formally holds the unpaired electron of the radical. These $d(\mathrm{~N}-$ $\mathrm{N})$ distances are displayed in Figure 2.

${ }^{\mathrm{b}}$ This distance corresponds to the closest pair of interstack radicals found in the 1-300K structure. 


\section{References}

${ }^{1}$ O. Kahn, C.J. Martinez, Science 1998, 279, 44-48

2 O. Sato, Nat. Chem. 2016, 8, 644-656.

${ }^{3}$ Spin Crossover in Transition Metal Compounds I-III, P. Gütlich, and H. A. Goodwin, Ed. Top. Curr. Chem., Springer-Verlag, Berlin, 2004, vol. 233-235.

${ }^{4}$ Spin-Crossover Materials-Properties and Applications; M.A. Halcrow, Ed.; John Wiley \& Sons: Hoboken, NJ, 2013.

${ }^{5}$ S. Brooker, Chem. Soc. Rev. 2015, 44, 2880-2892.

${ }^{6}$ T.M. Barclay, A.W. Cordes, N.A. George, R.C. Haddon, M.E. Itkis, M.S. Mashuta, R.T. Oakley, G.W. Patenaude, R.W. Reed, J.F. Richardson, H. Zhang, J. Am. Chem. Soc. 1998, 120, 352360.

${ }^{7}$ W. Fujita, K. Awaga, Science 1999, 286, 261-262.

${ }^{8}$ D.A. Shultz, R.M. Fico Jr., P.D. Boyle, J.W. Kampf, J. Am. Chem. Soc. 2001, 123, 1040310404.

${ }^{9}$ M.E. Itkis, X. Chi, A.W. Cordes, R.C. Haddon, Science 2002, 296, 1443-1445.

${ }^{10}$ J.L. Brusso, O.P. Clements, R.C. Haddon, M.E. Itkis, A.A. Leitch, R.T. Oakley, R.W. Reed and J.F. Richardson, J. Am. Chem. Soc. 2004, 126, 8256-8265. 
11 J.L. Brusso, O.P. Clements, R.C. Haddon, M.E. Itkis, A.A. Leitch, R.T. Oakley, R.W. Reed and J.F. Richardson, J. Am. Chem. Soc. 2004, 126, 14692-14693.

${ }^{12}$ K. Lekin, S.M. Winter, L.E. Downie, X.Z. Bao, J.S. Tse, S. Desgreniers, R.A. Secco, P.A. Dube and R.T. Oakley, J. Am. Chem. Soc. 2010, 132, 16212-16224.

${ }^{13}$ S. Matsumoto, T. Higashiyama, H. Akutsu, S. Nakatsuji, Angew. Chem. Int. Ed. 2011, 50, 10879-10883.

${ }^{14}$ R.G. Hicks, Nat. Chem. 2011, 3, 189-191.

${ }^{15}$ H. Phan, K. Lekin, S.M. Winter, R.T. Oakley and M. Shatruk, J. Am. Chem. Soc. 2013, 135, $15674-15677$.

${ }^{16}$ K. Lekin, H. Phan, S.M. Winter, J.W.L. Wong, A.A. Leitch, D. Laniel, W. Yong, R.A. Secco, J.S. Tse, S. Desgreniers, P.A. Dube, M. Shatruk and R.T. Oakley, J. Am. Chem. Soc. 2014, 136, 8050-8062.

${ }^{17}$ T. Li, G. Tan, D. Shao, J. Li, Z. Zhang, Y. Song, Y. Sui, S. Chen, Y. Fang and X. Wang. J. Am. Chem. Soc. 2016, 138, 10092-10095.

${ }^{18}$ J.M. Rawson, A. Alberola, A. Whalley, J. Mater. Chem. 2006, 16, 2560-2575.

${ }^{19}$ J.M. Rawson, J.J. Hayward, in Spin-Crossover Materials: Properties and Applications, ed. Halcrow, M.A. Wiley, Oxford, 2013, pp. 225-237.

${ }^{20}$ A. Alberola, J. Burley, R.J. Collis, R.J. Less, J.M. Rawson, J. Organomet. Chem. 2007, 692, 2750-2760. 
${ }^{21}$ A. Alberola, R.J. Collis, S.M. Humphrey, R.J. Less, J.M. Rawson, Inorg. Chem. 2006, 45, 1903-1905.

${ }^{22}$ A. Alberola, D.J. Eisler, L. Harvey, J.M. Rawson, CrystEngComm 2011, 13, 1794-1796.

${ }^{23}$ R. Suizu, A. Iwasaki, Y. Shuku, K. Awaga, J. Mater. Chem. C 2015, 3, 7968-7977.

${ }^{24}$ E.M. Fatila, R.A. Mayo, M. Rouzières, M.C. Jennings, P. Dechambenoit, D.V. Soldatov, C. Mathonière, R. Clérac, C. Coulon, K.E. Preuss, Chem. Mater. 2015, 27, 4023-4032.

${ }^{25}$ G.D. McManus, J.M. Rawson, N. Feeder, E.J.L. Mclnnes, J.J. Novoa, R. Burriel, F. Palacio, P. Oliete, J. Mater. Chem. 2001, 11, 1992-2003.

${ }^{26}$ W. Fujita, K. Awaga, H. Matsuzaki, H. Okamoto, Phys. Rev. B 2002, 65, 064434/1- 064434/9

${ }^{27}$ H. Matsuzaki, W. Fujita, K. Awaga, H. Okamoto, Phys. Rev. Lett. 2003, 91, 017403.

${ }^{28}$ P. Naumov, J.P. Hill, K. Sakurai, M. Tanaka, K. Ariga, J. Phys. Chem. A 2007, 111, 64496455

${ }^{29}$ T. Kon, S. Oguri, I. Katayama, T. Kodaira, J. Takeda, Phys. Rev. B 2009, 79, 035106/1035106/4

${ }^{30}$ I. Katayama, T. Kon, K. Mitarai, J. Takeda, Phys. Rev. B 2009, 80, 092103/1-092103/4.

${ }^{31}$ K. Ohno, Y. Noguchi, T. Yokoi, S. Ishii, J. Takeda, M. Furuya, ChemPhysChem, 2006, 7, 1820-1824.

${ }^{32}$ C.S. Clarke, J. Jornet-Somoza, F. Mota, J.J. Novoa, M. Deumal, J. Am. Chem. Soc. 2010, $132,17817-17830$ 
${ }^{33}$ A. Domingo, M. Vérot, F. Mota, C. de Graaf, J.J. Novoa, V. Robert, Phys. Chem. Chem. Phys. 2013, 15, 6982-6989.

${ }^{34}$ S. Vela, F. Mota, M. Deumal, R. Suizu, Y. Shuku, A. Mizuno, K. Awaga, M. Shiga, J.J. Novoa, J. Ribas-Arino, Nat. Commun. 2014, 5, 4411

${ }^{35}$ S. Vela, M. Deumal, M. Shiga, J.J. Novoa, J. Ribas-Arino, Chem. Sci. 2015, 6, 2371-2381.

${ }^{36}$ R. Car and M. Parrinello, Phys. Rev. Lett. 1985, 55, 2471-2474.

${ }^{37}$ M. Fumanal, S. Vela, J. Ribas-Arino, J.J. Novoa, Chem. Asian J. 2014, 9, 3612-3622.

${ }^{38}$ T.M. Barclay, A.W. Cordes, N.A. George, R.C. Haddon, R.T. Oakley, T.M.T. Palstra, G.W. Patenaude, R.W. Reed, J.F. Richardson, H. Zhang, Chem. Commun. 1997, 873-874.

${ }^{39}$ A. Alberola, O.P. Clements, R.J. Collis, L. Cubbitt, C.M. Grant, R.J. Less, R.T. Oakley, J.M. Rawson, R.W. Reed, C.M. Robertson, Cryst. Growth. Des. 2008, 8, 155-161.

${ }^{40}$ R.S. Mulliken, W.B. Person, Molecular Complexes; Wiley \& Sons: New York, 1969; Chapter 16.

${ }^{41}$ Z-h Cui, H. Lischka, H.Z. Beneberu, M. Kertesz, J. Am. Chem. Soc. 2014, 136, 5539-5542.

${ }^{42}$ M. Capdevila-Cortada, J.S. Miller, J.J. Novoa, Chem. Eur. J. 2015, 21, 6420-6432.

${ }^{43}$ (a) A.D. Becke, Phys. Rev. A, 1988, 38, 3098; (b) A.D. Becke, J. Chem. Phys., 1993, 98, 5648 
${ }^{44}$ (a) P.C. Hariharan, J.A. Pople, Theor. Chim. Acta, 1973, 28, 213; (b) M.M. Francl, W.J. Petro, W.J. Hehre, J.S. Binkley, M.S. Gordon, D.J. DeFrees, J.A. Pople, J. Chem. Phys., 1982, 77, 3654.

${ }^{45}$ Gaussian 09, Revision D.01, M. J. Frisch, G. W. Trucks, H. B. Schlegel, G. E. Scuseria, M. A. Robb, J. R. Cheeseman, G. Scalmani, V. Barone, B. Mennucci, G. A. Petersson, H. Nakatsuji, M. Caricato, X. Li, H. P. Hratchian, A. F. Izmaylov, J. Bloino, G. Zheng, J. L. Sonnenberg, M. Hada, M. Ehara, K. Toyota, R. Fukuda, J. Hasegawa, M. Ishida, T. Nakajima, Y. Honda, O. Kitao, H. Nakai, T. Vreven, J. A. Montgomery, Jr., J. E. Peralta, F. Ogliaro, M. Bearpark, J. J. Heyd, E. Brothers, K. N. Kudin, V. N. Staroverov, R. Kobayashi, J. Normand, K. Raghavachari, A. Rendell, J. C. Burant, S. S. Iyengar, J. Tomasi, M. Cossi, N. Rega, J. M. Millam, M. Klene, J. E. Knox, J. B. Cross, V. Bakken, C. Adamo, J. Jaramillo, R. Gomperts, R. E. Stratmann, O. Yazyev, A. J. Austin, R. Cammi, C. Pomelli, J. W. Ochterski, R. L. Martin, K. Morokuma, V. G. Zakrzewski, G. A. Voth, P. Salvador, J. J. Dannenberg, S. Dapprich, A. D. Daniels, Ö. Farkas, J. B. Foresman, J. V. Ortiz, J. Cioslowski, and D. J. Fox, Gaussian, Inc., Wallingford CT, 2009.

${ }^{46}$ (a) J.P. Perdew, K. Burke, M. Ernzerhof, Phys. Rev. Lett. 1996, 77, 3865-3868; (b) J.P. Perdew, K. Burke, M. Ernzerhof, Phys. Rev. Lett. 1997, 78, 1396.

${ }^{47}$ D. Vanderbilt, Phys. Rev. B 1990 41, 7892-7985.

${ }^{48}$ P. Giannozzi, S. Baroni, N. Bonini, M. Calandra, R. Car, C. Cavazzoni, D. Ceresoli, G.L. Chiarotti, M. Cococcioni, I. Dabo, A. Dal Corso, S. Fabris, G. Fratesi, S. de Gironcoli, R. Gebauer, U. Gerstmann, C. Gougoussis, A. Kokalj, M. Lazzeri, L. Martin-Samos, N. Marzari, F. Mauri, R. Mazzarello, S. Paolini, A. Pasquarello, L. Paulatto, C. Sbraccia, S. Scandolo, G. Sclauzero, A.P. Seitsonen, A. Smogunov, P. Umari and R.M. Wentzcovitch, J. Phys. Condens. Matter, 2009, 21, 395502. (Quantum ESPRESSO v. 4.2.1).

${ }^{49}$ S. Grimme, J. Comput. Chem. 2006, 27, 1787-1799. 
${ }^{50}$ T. Bucko, J. Hafner, S. Lebègue, J. Ángyán, J. Phys. Chem. A 2010, 114, 11814-11824.

${ }^{51}$ CPMD v. 3.13.2. copyright IBM Corp., 1990-2009; copyright MPI für Festkörperforschung Stuttgart, 1997-2001. www.cpmd.org

${ }^{52}$ G.J. Martyna, M.L. Klein, M. Tuckerman, J. Chem. Phys. 1992, 97, 2635-2643.

${ }^{53}$ R. L. Carlin, Magnetochemistry, Springer Science \& Business Media, 2012. 
TOC graphic<smiles>CN1Sc2cccc(C#N)c2S1</smiles>

Bistable Organic Radical

Diamagnetic Paramagnetic

Crystal Crystal

$\geqslant / 2 \underset{\substack{\text { 2nd order } \\ \text { transition }}}{\stackrel{\text { temp. }}{\rightleftarrows}} \geqslant$ 